complications. Warfarin dosage is age and weight dependent, so needs to be carefully monitored to achieve the correct dosing. The 2 year old has experienced symptoms of transient ischaemic attacks with an INR of 1.8 and extensive bruising with an INR $>2.7$. There is also the psychological and emotional impact involved with the frequent hospital admissions and blood tests. This is all in addition to the impact of the neurodisability, which has resulted from the initial thrombotic insult.

\section{G412(P) TO EVALUATE THE MANAGEMENT OF ACUTE PAINFUL CRISIS, OUTCOMES OF SAFETY AND EFFICACY OF CODEINE IN CHILDREN WITH SICKLE CELL DISEASE}

${ }^{1} 0$ Almossawi, ${ }^{2}$ O Wilkey. ${ }^{1}$ Pharmacy, North Middlesex University Hospital NHS Trust, London, UK; ${ }^{2}$ Paediatrics, North Middlesex University Hospital NHS Trust, London, UK

\subsection{6/archdischild-2015-308599.366}

Method A retrospective audit was performed on all admissions with diagnosis of sickle cell pain from January to December 2013. Case notes of 54 patients were reviewed using pre-specified audit criteria. Types of analgesics administered at home and in hospital and pain scores were recorded where available from the case notes.

Results Over the one-year period, 91 admissions were recorded. The average age was 6.6 years $( \pm 4.1)$, with the majority of children $(45 \%)$ aged between 1 and 5 years. The most prevalent haemoglobinopathy was HbSS (82\%). The majority of patients (61\%) had a single painful crisis admission, 33\% had 2-3 admissions, and the remainder presented with four or more admissions.

Most patients (93\%) received one or more analgesics prior to admission. The drug of choice varied, with paracetamol and ibuprofen been the most frequently utilised combination (43\%), while $22 \%$ received opioids (20\% had codeine). Similar to the pre-admission trends, a wide range of analgesics were utilised in hospital with the majority of patients receiving paracetamol and ibuprofen combination (43\%). The doses utilised at home were considerably less than those given on admission, suggesting inadequate pain management at home. Codeine was given in $37 \%$ of the cases in and it was observed that patients who received codeine had significantly higher initial pain scores on admission compared with those who did not receive codeine. However the administration of codeine did not greatly improve the pain scores and additional step up analgesia was required in $10 \%$ of cases who received codeine in hospital.

Conclusion Clear analgesic benefits could not be demonstrated for codeine use with regards to the outcome of efficacy. Although dihydrocodeine is now the weak opioid of choice at our unit added safety measures similar to those with codeine are in place. The audit was limited by poor documentation which have been addressed by the introduction of clearer updated local documents.

\section{G413(P) PROSPECTIVE STUDY OF PROCALCITONIN LEVELS IN CHILDREN WITH CANCER PRESENTING WITH FEBRILE NEUTROPENIA}

${ }^{1} V$ Hemming, ${ }^{2} \mathrm{~B}$ Phillips. ' General Paediatrics, Bradford Royal Infirmary, Bradford, UK; ${ }^{2}$ Paediatric Oncology, Leeds General Infirmary, Leeds, UK

10.1136/archdischild-2015-308599.367
Febrile neutropenia is an important cause of morbidity and mortality in children receiving treatment for cancer. Although cases of severe infection need inpatient treatment with broad-spectrum antibiotics there are a number of children with non-severe infection who are over treated. Previous meta-analysis showed further research is needed into the use of biomarkers, such as procalcitonin, as part of risk prediction in febrile neutropenia. No previous studies have looked at the use of biomarkers as an addition to existing clinical decision rules. Procalcitonin is currently being reviewed by NICE as part of it's diagnostics assessment programme.

Aims To determine if procalcitonin can be used to diagnose or exclude severe infection on presentation with febrile neutropenia and if it has additional benefit when used with existing clinical decision rules.

Method This is a prospective cohort study of a diagnostic test. Patients between birth and 18 years old were who were admitted to the paediatric oncology and haematology wards with febrile neutropenia were included. Blood was taken for a procalcitonin level at admission as well as routine investigations.

Results Forty-one episodes were included from 26 patients. Procalcitonin level of $>2 \mathrm{ng} / \mathrm{dL}$ has a likelihood ratio of $14.6[95 \%$ CI $1.8,120.4]$ and a sensitivity of $43 \%$ and specificity of $97 \%$. For none of the clinical decision rules did the procalcitonin odds ratio reach significance although all of the odds ratios were over one.

Conclusion This study does not show a benefit in using procalcitonin in febrile neutropenia. The cut off of $2 \mathrm{ng} / \mathrm{dL}$ has a high likelihood ratio for severe infection but poor sensitivity. There is no significant additional benefit of procalcitonin when used with existing clinical and laboratory features in clinical decision rules.

\section{G414(P) A RETROSPECTIVE ANALYSIS OF STEROID INDUCED BRADYCARDIA IN CHILDHOOD MALIGNANCY: CLINICAL RELEVANCE?}

${ }^{1} \mathrm{~N}$ Canfield, ${ }^{2} \mathrm{~V}$ Bharadwaj, ${ }^{2} \mathrm{R}$ Ramanujachar. ${ }^{1}$ Faculty of Medicine, University of Southampton, Southampton, UK; ${ }^{2}$ Paediatric Haematology-Oncology, Southampton Children's Hospital, University Hopsital Southampton NHS Foundation Trust, Southampton, UK

\subsection{6/archdischild-2015-308599.368}

Aims Determine incidence, clinical significance and management of steroid induced bardycardia in children undergoing cancer chemotherapy

Methodology Retrospective, observational, case note review of children treated at a tertiary paediatric oncology centre between $1^{\text {st }}$ January 2013 and $1^{\text {st }}$ September 2014 (21 months). Patients were subdivided into four groups based on steroid type, dose and indication.

- High dose dexamethasone $\left(10 \mathrm{mg} / \mathrm{m}^{2} /\right.$ day $)$

- Standard dose dexamethasone $\left(6 \mathrm{mg} / \mathrm{m}^{2} /\right.$ day $)$

- Non-Hodgkin Lymphoma prednisolone $\left(60 \mathrm{mg} / \mathrm{m}^{2} /\right.$ day $)$

- Other (variable dosing)

Simultaneous data collected on age, baseline heart rate (HR) at presentation, lowest HR on steroids, blood pressure (BP), ECG and intra-ocular pressure (IOP).

Statistics: $P$ values calculated

Bradycardia of $\geq 30 \%$ was considered significant as it correlated with a Paediatric Early Warning Score (PEWS) of 2. 